\title{
PRODUÇÃO DE VIGAS ESTRUTURAIS EM PERFIL “I” COM PAINÉIS DE MADEIRA RECONSTITUÍDA DE Pinus taeda L. E Eucalyptus dunnii Maiden
}

\author{
Alexandre de Luna Pedrosa*, Setsuo Iwakiri**, Jorge Luis Monteiro de Matos*** \\ *Eng. Florestal, M.Sc. \\ **Eng. Florestal, Dr., Depto. de Engenharia e Tecnologia Florestal da UFPR - setsuo@floresta.ufpr.br \\ ***Eng. Florestal, Dr., Depto. de Engenharia e Tecnologia Florestal da UFPR - jmatos@floresta.ufpr.br \\ Recebido para publicação: 07/10/2005 - Aceito para publicação: 26/12/2005
}

\begin{abstract}
Resumo
Produção de vigas estruturais em perfil "I" com painéis de madeira reconstituída de Pinus taeda L.e Eucalyptus dunnii Maiden. Este trabalho teve como objetivo avaliar o comportamento de vigas estruturais em perfil "I", constituídas com flanges de Pinus taeda e Eucalyptus dunnii e com almas de painéis OSB, compensados de Pinus taeda e Eucalyptus dunnii, produzidas na região Sul do Brasil. O experimento foi estruturado para seis tratamentos com cinco repetições, perfazendo um total de trinta vigas. As vigas foram montadas em laboratório com uso de aparato especial de prensagem, utilizando a resina resorcina-formaldeído. Os resultados dos ensaios de flexão estática demonstraram melhor comportamento das vigas compostas com flanges de Eucalipto em comparação às vigas com flanges de Pinus. Com relação às almas, as vigas compostas com painéis OSB apresentaram melhores resultados de MOE em comparação aos painéis compensados de Pinus e de Eucalipto. Para o MOR, não foram constatadas as mesmas tendências observadas para o MOE. A composição de vigas "I" com flange de Eucalipto e alma de OSB seria uma alternativa viável, sob o ponto de vista técnico e econômico.
\end{abstract}

Palavras-chave: Vigas estruturais; vigas com perfil "I"; compensados; painéis OSB.

\section{Abstract}

Production of "I - Joists" with wood panels of Pinus taeda L. and Eucalayptus dunnii Maiden. This research was developed to evaluate the performance of "I - joists" made out of LVL flanges of Pinus taeda and Eucalyptus dunnii, with webs of the OSB, plywoods of Pinus taeda and Eucalyptus dunnii, manufactured in south region of Brazil. The experimental chart was defined for six treatments with five repetitions, totalizing thirty "I - joists". The "I - joists" were manufactured in laboratory through special apparatus for pressing, using the resorcina-formaldehyde resin. The results of bending strength tests showed the best performance of "I - joists" made with flanges of Eucalipto in comparison to flanges of Pinus. In relation to webs, the "I - joists" made with OSB showed the highest mean value of MOE in comparison to plywood of Pinus and Eucalipto species. For the MOR, it were not observed the same tendency obtained to MOE. The "I - joists" made with flange of Eucalipto and web of OSB would be a technical and economically feasible alternative.

Keywords: Structural lumber; "I - joists"; plywood; OSB.

\section{INTRODUÇÃO}

O uso de painéis de madeira reconstituída em substituição à madeira sólida tem ganhado destaque nas últimas décadas em função da necessidade de otimização do uso da madeira e pelas características tecnológicas de produtos colados de madeira. Suas aplicações não se limitam apenas à fabricação de móveis, embalagens e como componentes estruturais de paredes, pisos e coberturas de edificações, mas também como elementos estruturais "engenheirados" - com destaque a vigas em perfil "I".

A utilização de espécies provenientes de florestas plantadas tem uma contribuição importante, reduzindo a pressão sobre a utilização de espécies de florestas nativas. O rápido crescimento de espécies plantadas, como Pinus e Eucalipto, atendem à demanda crescente e necessidade por grandes volumes de 
madeira para suprimento das indústrias madeireiras. As densidades de médias a baixas das espécies de florestas plantadas deixam de ser restrições para uso estrutural, através do emprego de tecnologia de colagem e processos de fabricação de produtos de madeira reconstituída.

De acordo com Marra (1992), os produtos reconstituídos de madeira são fabricados através da colagem de lâminas, tábuas, sarrafos, partículas ou fibras com dimensões e características definidas em função de suas aplicações finais. $\mathrm{O}$ balanço estrutural do produto é conferido através da ligação adesiva entre os elementos de madeira, sua distribuição e orientação na estrutura do composto. A Associação de Madeira Engenheirada dos Estados Unidos divide esses produtos em quatro categorias: (i) a de painéis estruturais, que incluem os compensados e painéis de partículas orientadas - OSB (Oriented Strand Board); (ii) a de madeira laminada colada (MLC, Glulam); (iii) a de madeira composta estrutural, em que se enquadram os painéis de lâminas paralelas ou laminated veneer lumber (PLP, LVL); e (iv) a das vigas com perfil "I" (Engineered Wood Association, 2002).

Componentes estruturais eficientes podem ser produzidos pela combinação de painéis estruturais através da colagem. Um dos elementos com desenho mais eficiente são as vigas com seção em forma de "I", que apresenta boa distribuição das tensões com economia de material (Forest Products Laboratory, 1999).

O surgimento das vigas de madeira com seção "I" data de 1969, e o seu desenvolvimento foi originalmente motivado pela performance e não pelo preço. Projetos arquitetônicos da época, inspirados por consumidores que desejavam vãos de maiores extensões em seus ambientes, necessitavam de elementos nos pavimentos superiores mais resistentes que as peças de madeira serradas dentro de limitações dimensionais. Inicialmente foram produzidas vigas "I" com grandes almas de compensado e flanges de madeira serrada de menores dimensões, que dessa forma possibilitavam a obtenção de um maior número de peças livres de defeitos para utilização (Fisette, 2000). Gramola (1992) ressalta as vantagens econômicas e ambientais que a otimização e racionalização do sistema estrutural em forma de "I" trariam às estruturas de madeira. No entendimento dos projetistas das vigas "I", o desenho da seção é favorável à economia do material, representada pelas pequenas espessuras das almas, uma vez que a distribuição das maiores intensidades de tensões sobre a altura das vigas se dá nas extremidades, onde estão posicionadas as flanges (Fisette, 2000). Ainda de acordo com o autor, a forma da seção "I" proporciona uma relação resistência-peso muito favorável, além da economia do material.

As flanges podem ser tanto de madeira sólida como de painéis de lâminas paralelas (PLP, LVL). Atualmente, o PLP é mais amplamente empregado, tendo em vista as vantagens decorrentes da tecnologia de colagem de lâminas e otimização do uso de madeira, contando com apelo ambiental mais forte nos últimos anos. A resistência das flanges tem grande importância, por constituírem as extremidades da viga, onde, na parte superior, ocorre a maior tensão de compressão, enquanto que, na inferior, verificam-se tensões máximas de tração (Fisette, 2000).

A alma é normalmente constituída de painéis estruturais, como é o caso do compensado e OSB, com espessura mínima de $3 / 8$ pol. $(9 \mathrm{~mm})$, segundo as normas de padronização desses materiais (Engineered Wood Association, 2002). O posicionamento vertical da alma é responsável pelo alcance da altura na viga, um dos fatores de maior importância na relação entre as dimensões e a resistência à deflexão, entre outras características. O correto dimensionamento da relação entre a altura da viga e a distância entre os pontos de apoio é responsável também pela economia de material citada anteriormente.

Tanto os componentes quanto as vigas devem ser fabricados através de colagem com adesivos de uso exterior, como o fenol-formaldeído e a resorcina-formaldeído, sendo esta a mais indicada na montagem das vigas, devido a sua capacidade de cura a frio (Engineered Wood Association, 2002).

Este trabalho foi desenvolvido com o objetivo de produzir e avaliar o desempenho estrutural de vigas em perfil "I" constituídas de compensado estrutural, painéis de lâminas paralelas e painéis OSB, de madeira de Pinus taeda L. e Eucalyptus dunnii Maiden.

\section{MATERIAIS E MÉTODOS}

Foram utilizadas nesta pesquisa, madeiras de Pinus taeda provenientes do Município de Rio Negrinho (SC) e de Eucalyptus dunnii proveniente da região de Três Barras (SC). Os painéis OSB para composição da alma de vigas "I" foi obtido da linha de produção da empresa MASISA do Brasil, localizada em Ponta Grossa (PR). O adesivo utilizado na fabricação de painéis compensados e painéis de lâminas paralelas foi o fenol-formaldeído, e para vigas "I" foi utilizada a resorcina-formaldeído. 
As lâminas para fabricação de painéis de lâminas paralelas (PLP) foram processadas com espessura de 3,2 $\mathrm{mm}$, e para compensado, com 2,2 $\mathrm{mm}$. Após a secagem ao conteúdo de umidade de 6 a $8 \%$, as lâminas foram classificadas e separadas de acordo com o padrão de qualidade " $\mathrm{C}+$ ", conforme as especificações exigidas pelo Programa Nacional de Qualidade da Madeira (PNQM - ABIMCI).

Os painéis PLP de Pinus taeda e Eucalyptus dunnii foram fabricados com 11 camadas e espessura de $32 \mathrm{~mm}$, e os painéis compensados, com 5 camadas e $9 \mathrm{~mm}$ de espessura. O adesivo fenolformaldeído foi aplicado nas duas faces das lâminas pares, com gramatura de $400 \mathrm{~g} / \mathrm{m}^{2}$. Os painéis foram prensados nas seguintes condições: pressão específica de $10 \mathrm{kgf} / \mathrm{cm}^{2}$, temperatura de $135^{\circ} \mathrm{C}$ e tempo de prensagem de 25 minutos para PLP e de 9 minutos para compensados. Antes da fabricação das vigas, foram retiradas amostras para determinação da massa específica aparente dos painéis.

Posteriormente, os painéis compensados, PLP e OSB, foram seccionados numa serra circular esquadrejadeira em dimensões preestabelecidas de flanges e almas, para composição de vigas "I". As flanges, em número de 60 , foram obtidas com espessura de $32 \mathrm{~mm}$, largura de $38,1 \mathrm{~mm}$ e comprimento de $2.440 \mathrm{~mm}$, além de canaletas com $9 \mathrm{~mm}$ de largura e $10 \mathrm{~mm}$ de profundidade, para encaixe das almas. As 30 peças para composição das almas foram obtidas com espessura de $9 \mathrm{~mm}$, largura de 13,6 mm e comprimento de $2.440 \mathrm{~mm}$. Na tabela 1 está apresentado o plano experimental para produção das vigas "I".

Tabela 1. Plano experimental.

Table 1. Experimental chart.

\begin{tabular}{lcc}
\hline Tratamentos & Flanges & Almas \\
\hline T1 & PLP - Painéis de lâminas paralelas Pinus & OSB - Oriented Strand Board \\
T2 & CP - Compensado Pinus \\
T3 & CE - Compensado Eucalipto \\
\hline T4 & OSB - Oriented Strand Board \\
T5 & PLP - Painéis de lâminas paralelas Eucalipto & CP - Compensado Pinus \\
T6 & CE - Compensado Eucalipto \\
\hline
\end{tabular}

Para a produção das vigas "I", foi utilizada a composição com 100 partes de resina resorcinaformaldeído e 20 partes de endurecedor. O adesivo foi aplicado com uso de pincel nas duas superfícies a serem coladas, das almas e das flanges, com gramatura de $400 \mathrm{~g} / \mathrm{m}^{2}$. A prensagem foi realizada por meio de mecanismos de barras roscadas sobre toda extensão das vigas, distantes não mais que $30 \mathrm{~cm}$ uns dos outros. Nos entremeios dos mecanismos de prensagem, foram feitas amarras com tiras de aço tencionadas para que as vigas permanecessem prensadas durante a cura, num período de 10 a 15 horas sob temperatura ambiente (Figura 1). As vigas foram mantidas amarradas por um período de 4 dias, até o início dos testes.

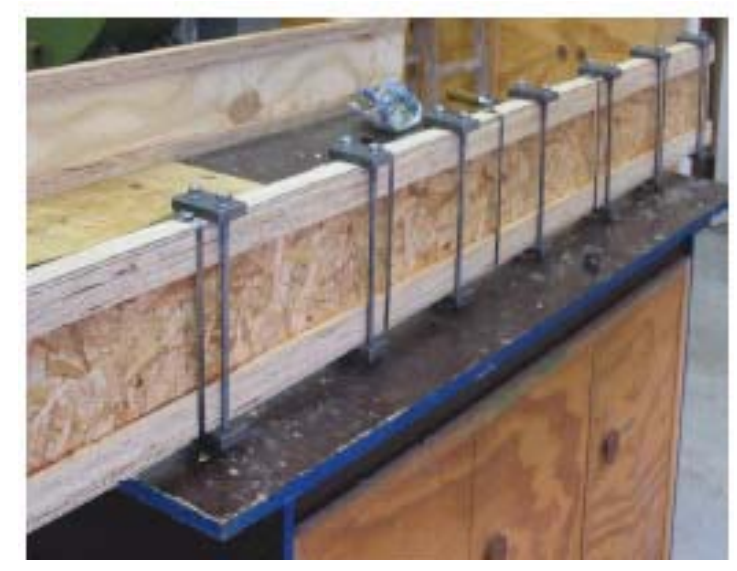

Figura 1. Prensagem e amarração das vigas "I".

Figure 1. Pressing and fastening of "I - joists". 
Após a colagem, as vigas ficaram com a altura de $180 \mathrm{~mm}$, conforme ilustrado na figura 2. Foram produzidas 5 vigas por tratamento, perfazendo um total de 30 vigas.

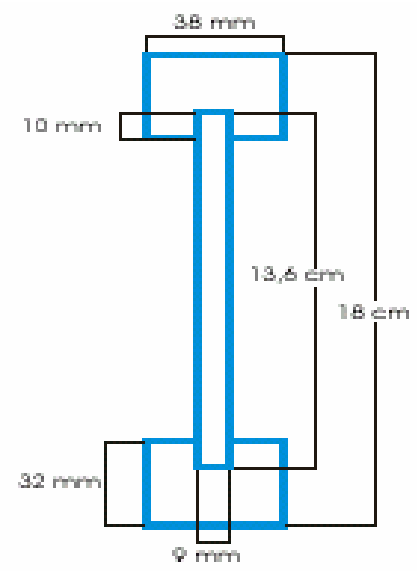

Figura 2. Desenho da seção da viga "I".

Figure 2. Design of "I - joist" cross section.

Os ensaios de flexão estática das vigas foram realizados em máquina universal de ensaios com capacidade para 30 toneladas (Figura 3), com base nos procedimentos descritos na norma ASTM D-198. Para cada viga, foram determinados os módulos de elasticidade e de ruptura à flexão.

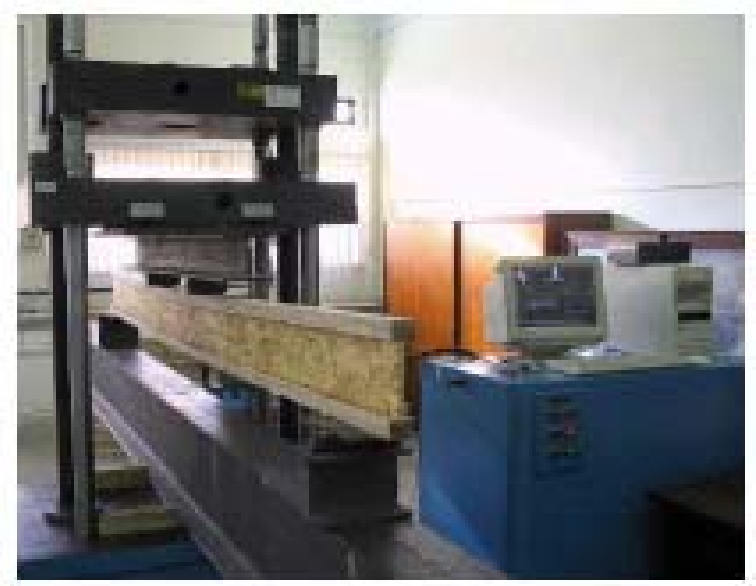

Figura 3. Ensaio de flexão estática das vigas "I".

Figure 3. Static bending test of "I - joist".

\section{RESULTADOS E DISCUSSÃO}

\section{Densidade dos painéis de madeira}

Os valores médios de densidade dos painéis de madeira utilizados para produção de vigas laminadas são apresentados na tabela 2.

Os painéis compensados e PLP de Eucalipto apresentaram maiores densidades em comparação com os painéis de Pinus. Essa diferença pode ser atribuída à maior densidade da madeira de Eucalipto em relação à madeira de Pinus e à ausência do fator de compactação durante a prensagem, ao contrário do que ocorre em painéis particulados e de fibras. 
Tabela 2. Densidade média dos painéis de madeira.

Table 2. Average density of wood panels.

\begin{tabular}{lcc}
\hline Tipos de Painéis & Densidade média $\left(\mathbf{g} / \mathbf{c m}^{\mathbf{3}}\right)$ & Coeficiente de variação (\%) \\
\hline PLP de Pinus & 0,595 & 3,54 \\
PLP de Eucalipto & 0,776 & 2,57 \\
Compensado de Pinus & 0,559 & 3,05 \\
Compensado de Eucalipto & 0,683 & 1,91 \\
OSB de Pinus & 0,630 & 2,59 \\
\hline
\end{tabular}

PLP: painéis de lâminas paralelas; OSB: painéis de partículas orientadas.

A densidade média de $0,630 \mathrm{~g} / \mathrm{cm}^{3}$ dos painéis OSB de Pinus está dentro do padrão industrial estabelecido pela empresa MASISA. Para os painéis particulados, a densidade pode ser controlada através da quantidade de partículas de madeira e grau de compactação desse material no processo de prensagem.

\section{Módulo de elasticidade e módulo de ruptura das vigas "I"}

Os valores médios de módulo de elasticidade (MOE) e módulo de ruptura (MOR) das vigas "I" estão apresentados na tabela 3 e ilustrados nas figuras 4 e 5.

Tabela 3. Valores médios de módulo de elasticidade (MOE) e de ruptura (MOR) das vigas "I". Table 3. Average values of modulus of elasticity and rupture of "I - joists".

\begin{tabular}{lcccc}
\hline Tratamentos / Repetições & Flanges & Almas & MOE $\mathbf{( k g f / \mathbf { c m } ^ { 2 } )}$ & MOR $\left(\mathbf{k g f} / \mathbf{c m}^{2}\right)$ \\
\hline T1 / 5 & PLP Pinus & OSB & $136.160 \mathrm{~b}$ & $929 \mathrm{a}$ \\
T2 / 5 & PLP Pinus & CP & $120.445 \mathrm{~b}$ & $976 \mathrm{a}$ \\
T3 / 5 & PLP Pinus & CE & $104.167 \mathrm{~b}$ & $831 \mathrm{a}$ \\
T4 / 5 & PLP Eucalipto & OSB & $196.207 \mathrm{a}$ & $981 \mathrm{a}$ \\
T5 / 5 & PLP Eucalipto & CP & $141.723 \mathrm{~b}$ & $1.034 \mathrm{a}$ \\
T6 / 5 & PLP Eucalipto & CE & $132.607 \mathrm{~b}$ & $1.037 \mathrm{a}$ \\
\hline PLP: painéis.
\end{tabular}

PLP: painéis de lâminas paralelas; OSB: painéis de partículas orientadas; CP: compensado de Pinus; CE: compensado de Eucalipto. Médias seguidas de mesma letra dentro da coluna são estatisticamente iguais ao nível de probabilidade de $95 \%$.

As comparações entre as médias dos tratamentos T1 e T4, T2 e T5, T3 e T6, apresentados na tabela 3 e figuras 4 e 5, indicam claramente a influência das flanges no MOE e MOR das vigas. Embora apenas o tratamento T4 tenha apresentado MOE estatisticamente superior em relação aos demais tratamentos, em termos de médias absolutas, a utilização da flange de Eucalipto confere às vigas maiores valores de MOE e MOR em comparação às vigas produzidas com flange de Pinus. Esse resultado comprova a importância do material utilizado como flange na resistência das vigas "I", nas quais as partes submetidas às maiores tensões estão localizadas nas faces externas da viga. Portanto, a utilização de madeira com maior densidade e resistência mecânica pode contribuir de forma significativa na performance das vigas estruturais em perfil "I".

Com relação às almas de OSB, compensados de Pinus e de Eucalipto não foram constatadas diferenças estatísticas significativas para as médias do MOR das vigas "I". Por outro lado, as vigas compostas com flange de Eucalipto e alma de OSB apresentaram valor médio de MOE estatisticamente superior em comparação aos demais tratamentos. Tanto para vigas com flanges de Eucalipto quanto para as de Pinus, os resultados em termos de médias absolutas indicam uma tendência para maiores valores de MOE das vigas compostas com almas de OSB em relação aos compensados de Pinus e de Eucalipto. Para o MOR, não foi constatada a mesma tendência observada para o MOE.

Os resultados deste estudo mostram a viabilidade técnica da utilização de almas de OSB na produção de vigas em perfil "I'. É importante ressaltar também as vantagens econômicas do menor custo de painéis OSB em comparação aos painéis compensados, decorrentes de fatores como a tecnologia de produção automatizada, maior escala de produção e menor exigência em termos de qualidade das toras. 


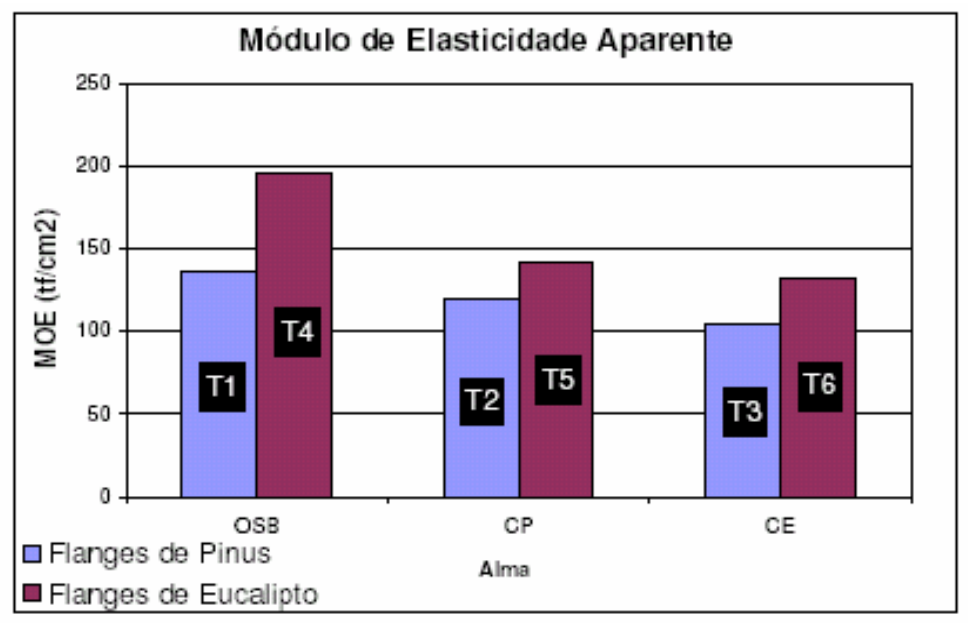

Figura 4. Histograma do módulo de elasticidade das vigas "I" $\left(\mathrm{tf} / \mathrm{cm}^{2}\right)$.

Figure 4. Hystogram of modulus of elasticity of "I - joists" $\left(\mathrm{tf} / \mathrm{cm}^{2}\right)$.

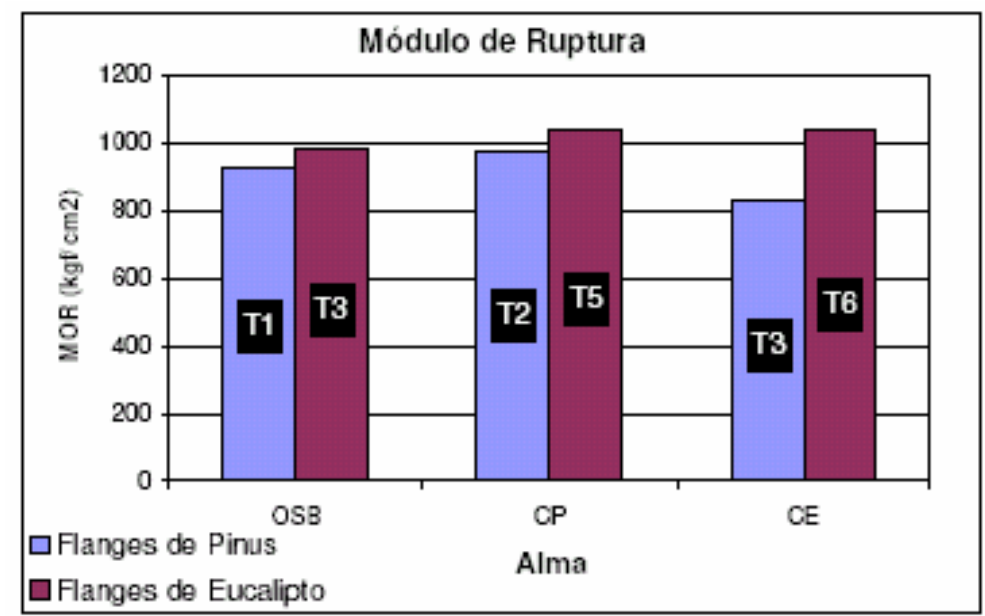

Figura 5. Histograma do módulo de ruptura das vigas "I" (tf $\left./ \mathrm{cm}^{2}\right)$.

Figure 5. Hystogram of modulus of rupture of "I- joists" $\left(\mathrm{tf} / \mathrm{cm}^{2}\right)$.

\section{CONCLUSÕES}

- As vigas em perfil "I" compostas de flange de Eucalipto apresentaram maiores valores médios em comparação às vigas com flange de Pinus.

- As vigas em perfil "I" compostas com almas de painéis OSB apresentaram maiores valores médios de MOE em relação às almas de compensado de Pinus e de Eucalipto.

- Para o MOR, não foi constatada uma tendência que possa definir a indicação de melhor tipo de painel para composição da alma de viga em perfil "I".

- Os resultados permitem concluir que a flange é o componente mais importante na composição de vigas em perfil "I".

- No conjunto de fatores técnico e econômico, o painel OSB é um produto mais adequado em comparação aos painéis compensados de Pinus e de Eucalipto para compor a alma de vigas em perfil "I". 


\section{REFERÊNCIAS}

AMERICAN SOCIETY FOR TESTING AND MATERIALS. Standard methods of testing structural panels in flexure - D 198. In: ANNUAL Book of ASTM Standards. Philadelphia, USA, 1994.

ENGINEERED WOOD ASSOCIATION. Engineered Wood Systems. Understanding Engineered Wood Products. Disponível em <http://www.apawood.org/woodu/secure/101/unit one/1unit1 sotry.cfm>

FISETTE, P. The Argument for I-joists: New products and increased competition make superior I-joist performance available at solid-lumber prices. Fine Homebuilding Mafazine, n. 129, p. 70-73. 2000.

FOREST PRODUCTS LABORATORY. Wood Handbook: wood as an engineering material. Madison, Wisconsin: United States Department of Agriculture, Forest Service, 1999. 463p.

GRAMOLA, D. S. Systems approach to design. In: FOREST PRODUCTS SOCIETY. Wood Products for Engineered Structures: Issues affecting growth and acceptance of engineered wood products. Madison, Wis.: Forest Products Society, 1992. 220p.

MARRA, A. A. Technology of Wood Bonding: principles and practice. New York: Van Nostrand Reinhold, 1992. 453p. 